\title{
ON THE ESTIMATION AND INTERPRETATION OF SENTINEL-1 TOPS INSAR COHERENCE
}

\author{
Urs Wegmüller $^{(1)}$, Maurizio Santoro ${ }^{(1)}$, Charles Werner ${ }^{(1)}$ and Oliver Cartus $^{(1)}$ \\ (1) Gamma Remote Sensing, Worbstrasse 225, 3073 Gümligen BE, Switzerland \\ tel: ++41 31951 7005,fax: ++4131951 7008, e-mail: wegmuller@gamma-rs.ch
}

\begin{abstract}
Sentinel-1 IWS acquisitions can be used for SAR interferometry. In our contribution the focus is on the S1 IWS InSAR coherence. For this we address in a first part the interferogram generation and coherence estimation. Then in a second part we use data examples to get a first impression on the potential of the S1 IWS InSAR coherence for landuse classification and to monitor temporal change
\end{abstract}

\section{INTRODUCTION}

In the 1990's ERS-1 C-band data acquired with short 3-day repeat intervals and after the launch of ERS2 also ERS-1/2 Tandem acquisitions with only one day interval permitted developing a range of applications based on the interferometric coherence, such as land use characterization [1] and the retrieval of vegetation parameters [2,3]. While a significant potential was found for the C-band data acquired with 1 and 3 day intervals, the potential was much lower with the 35-day repeat interval of the ENVISAT mission. With the launch of Sentinel-1A, C-band data with short intervals (12 days) are available. In combination with Sentinel$1 \mathrm{~B}$ the interval will be 6 days, available. It is therefore of interest to consider again more closely the interferometric coherence as a parameter for a range of thematic mapping applications.

In Section 2 we discuss aspects related to the coherence estimation. Then we show preliminary Sentinel-1 IWS results related to landuse classification (Section 3), and temporal change monitoring (Section 4).

\section{SENTINEL-1 IWS COHERNCE ESTIMATION}

InSAR processing of Sentinel-1 interferometric wide-swath (IWS) mode data acquired in the TOPS mode [4] is challenging. The steep azimuth spectrum ramp in each burst results in very stringent coregistration requirements [5]. Our existing coregistration method that considers also the scene topography was updated, so that it is applicable to the TOPS mode data. The main geometric transformation function is derived based on the orbit and surface geometry. For this a small correction (single offset in range and azimuth) is applied. This offset is typically determined using first an intensity cross-correlation and then a spectral diversity method [6] considering the InSAR phase differences in the along-track burst overlap regions.

The co-registered TOPS SLC pairs and stacks are then used to calculate a differential interferogram and estimate the interferometric coherence. Compared to ERS data, the resolution of the Sentinel-1 TOPS data is significantly better in range direction and significantly lower in azimuth direction. Applying a stronger multilooking in range instead of in azimuth direction results in Sentinel-1 interferograms with a comparable spatial resolution and number of looks as for ERS or ENVISAT. Major advantages of Sentinel-1 compared to these sensors are the much larger spatial coverage and the availability of coherence also at cross-polarization.

\section{S1 IWS COHERENCE PRODUCTS}

Using ERS data it was shown that C-band coherence over short time intervals (1-day, 3-days) contains information on landuse classes such as forest, water, urban areas and others [1-3]. To get an idea of the related potential of Sentinel-1A data, we generated examples of "coherence products" consisting of the interferometric coherence, the average backscatter, and the backscatter change between the two scenes of the interferometric pair. Figure 1 shows coherence products over an area including Mexico City, the large urban area visible in the sub-swath to the East, with 12, 24 and 48 days interval. Even with 12 days repeat interval the coherence is relatively low over vegetated areas (green color in Figure 1 indicates low coherence, i.e. a low red value in this RGB combination). Distinguishing forest and short vegetation (e.g. agricultural areas) appears to be critical. An RGB composite of the 12-, 24- and 48day coherence is shown in Figure 2. The brownish 


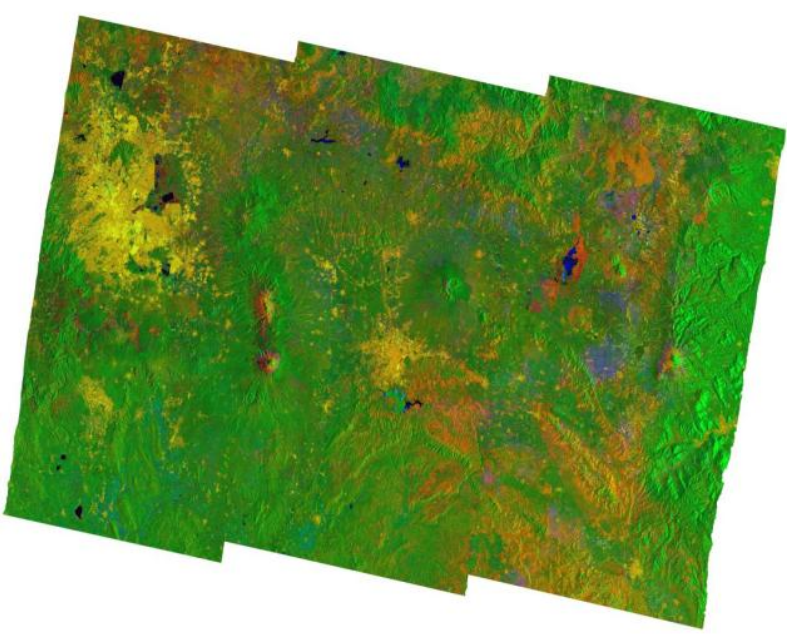

20141015 - 20141027 (12 days)

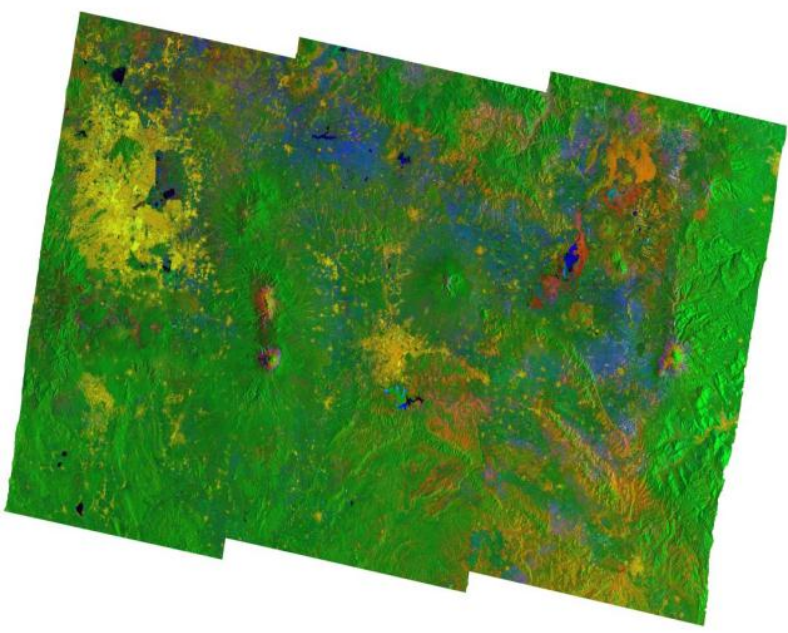

$20141015-20141108$ (24 days)

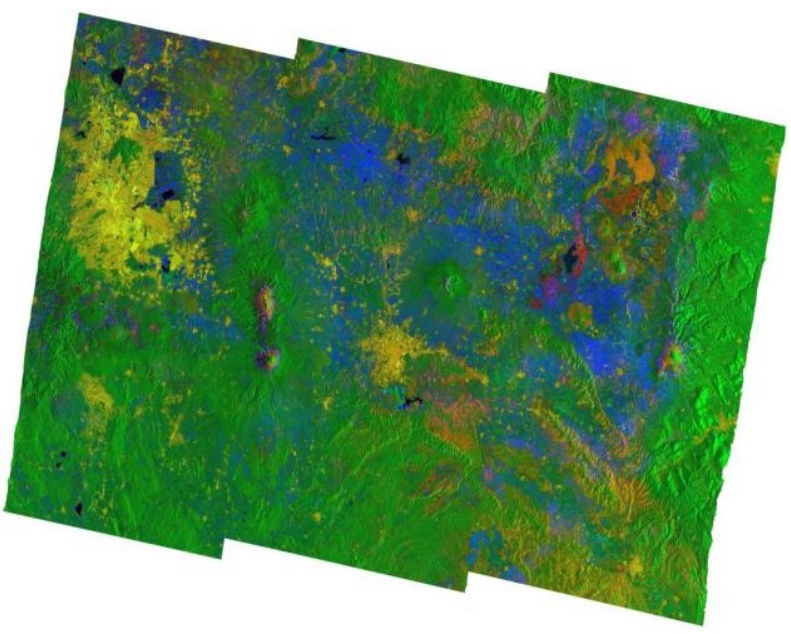

20141015 - 20141202 (48 days)

Figure 1 S1 IWS VV-Pol. coherence products over a region including Mexico City (large yellow patch). The coherence product is an RGB composite of the interferometric coherence (red), the average backscatter (green) and the backscatter change (blue).

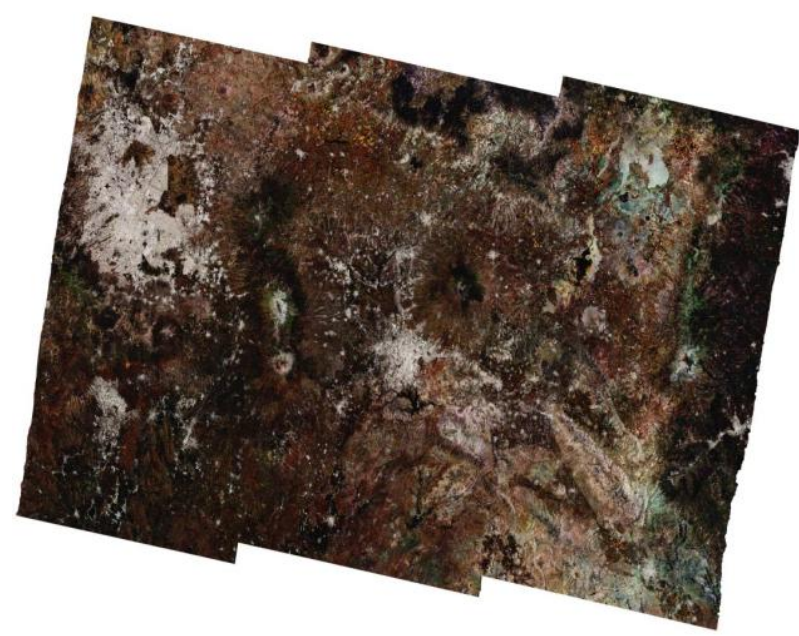

Figure 2 S1 IWS VV-pol. multi-temporal composite of the 3 coherence images of Figure 1 (Red: 20141015 20141027 (12 days), Green: 20141015 - 20141108 (24 days), Blue: 20141015 - 20141202 (48 days)
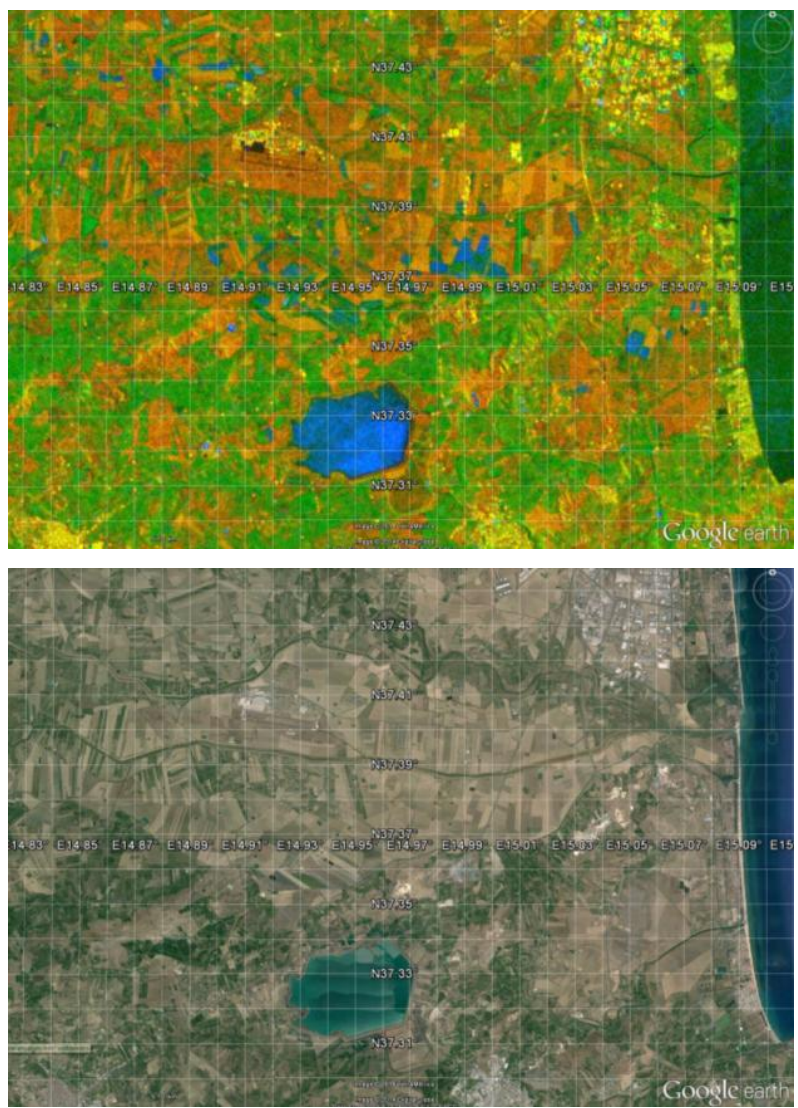

Figure 3 Small section of a Sentinel-1A VV-pol. coherence product (top) derived from IWS data acquired over Sicily on 20140809 and 20140821. For comparison an optical image acquired on 20130729, as found in Google Earth, is shown (bottom).

color present for much of the areas indicates that the coherence over lower vegetation was starting from a relatively low level for 12 days but it was further 
reduced with the increasing time intervals. Over the urban area the coherence remained high. A second example of IWS VV-pol coherence product is shown in Figure 3, a 12-day S1 IWS coherence product over an agricultural area in Sicily. The coherence is spatially varying over agricultural fields. High coherence levels at this time of the year are not unusual in a 12-days coherence product as many fields are already bare after harvesting.

High coherence values are observed over an arid area in Iraq in another 12-days coherence product (Figure 4). The coherence contrast between uncultivated and cultivated areas is large. Additionally, strong backscatter change is observed for some fields, most likely caused by irrigation or mechanical cultivation.
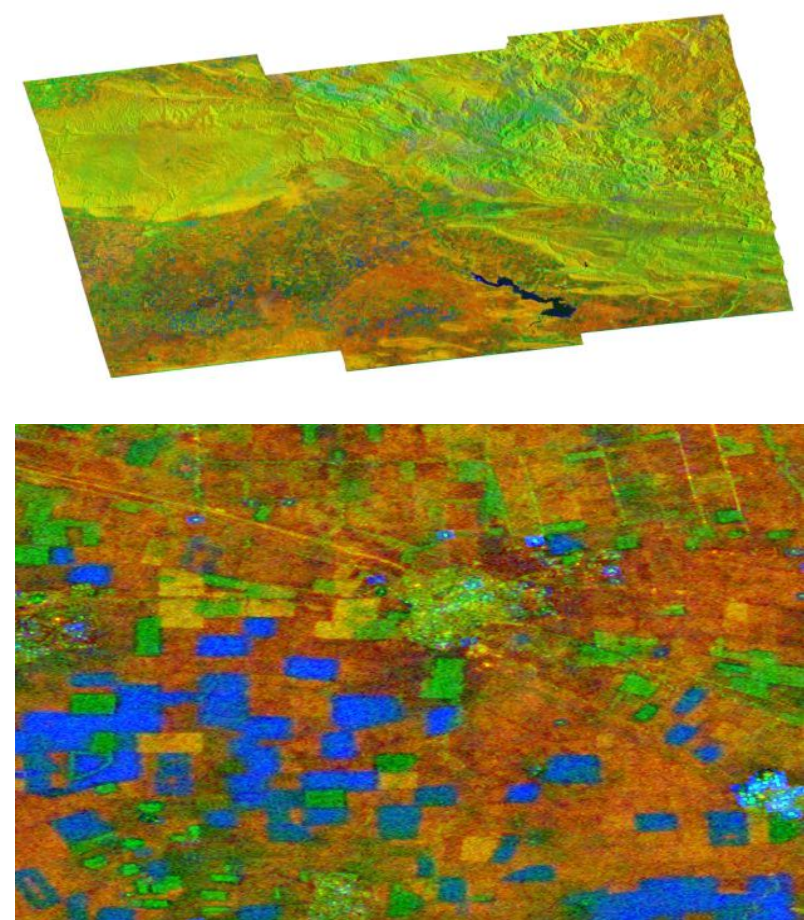

Figure 4 Sentinel-1A VV-pol. coherence product over an area in Iraq (top) derived from IWS data acquired on 20141003 and 20141015 and small section thereof over an agricultural area (bottom).

These results indicate that the C-band coherence is significantly lower over vegetation after 12 days (Sentinel-1 ) as compared to after 1 or 3 days (ERS). This is particularly the case for temperate regions as Switzerland (Figure 5). A November 12-days pair (which should be quite ideal to separate agricultural areas and forest) shows low coherence over many fields. The potential to separate low (agriculture, grassland) and high (forest) vegetation therefore appears to be limited. Hopefully, this potential will improve with the 6-da intervals that will become available after the launch of S1B in early 2016.
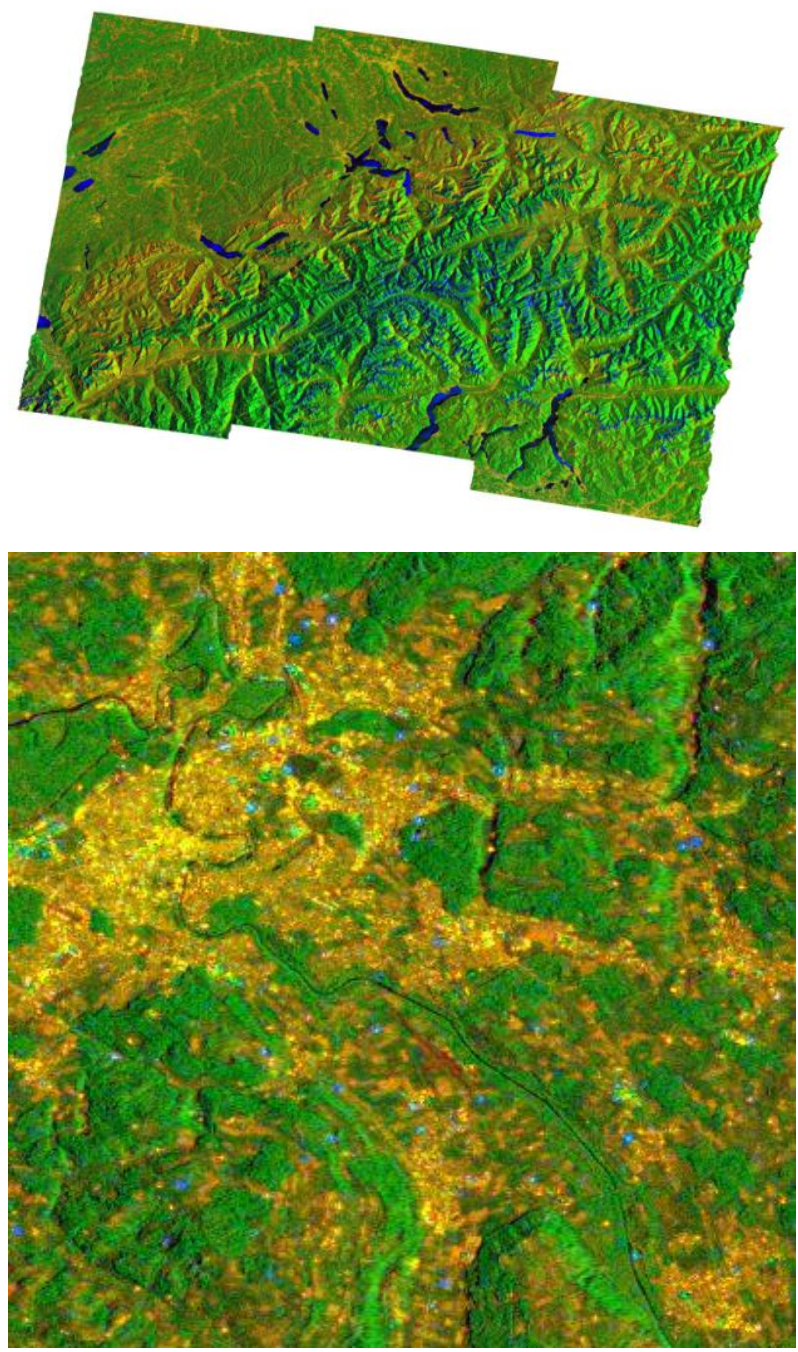

Figure 5 Sentinel-1A VV-pol. coherence product over Switzerland derived from IWS data acquired on 20141115 and 20141127 and small section thereof over Berne and suburbs (bottom).

\section{CHANG E MONITORING}

Coherence is also of interest for change monitoring For our initial investigation we used a stack of 12 IWS VV-pol. scenes over Mexico City. This time series provided 12-days repeat intervals between all subsequent acquisitions except between acquisitions 4 and 5 and between acquisitions 11 and 12. To assess the multi-temporal change of the coherence between subsequent acquisitions we generated 4 RGB composites, each one including the coherence of 3 


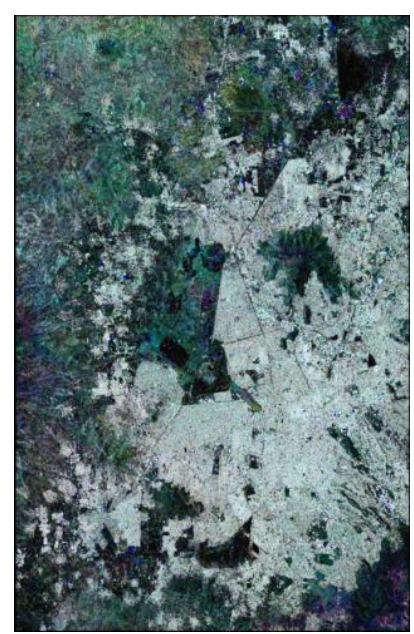

intervals $1,2,3$

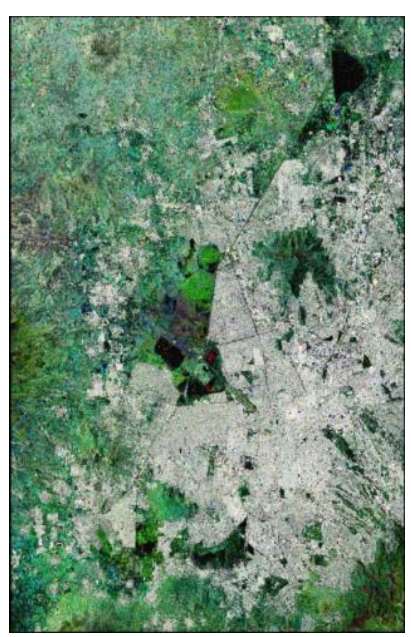

intervals $4,5,6$

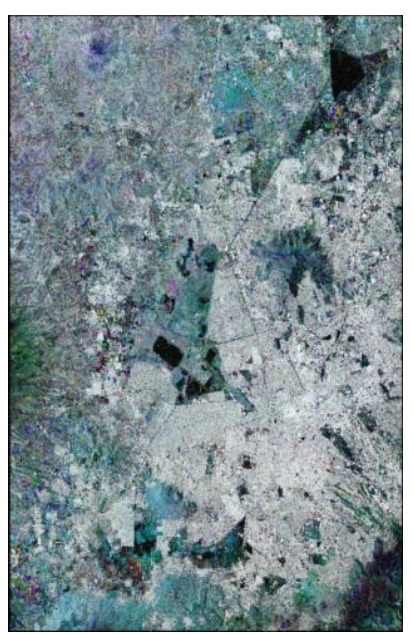

intervals $7,8,9$

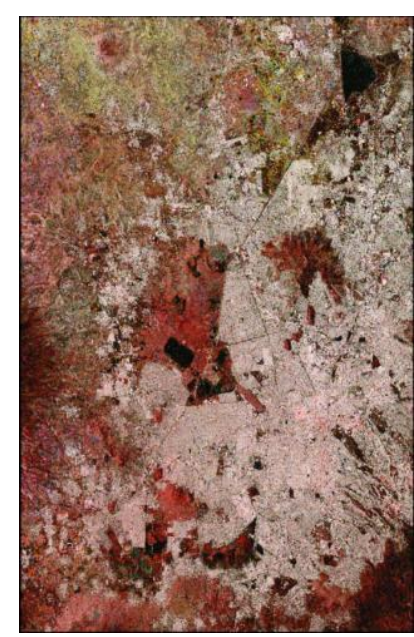

intervals $9,10,11$

Figure 6 Multi-temporal S1 IWS VV-pol. coherence RGB composites over Mexico City.

different periods. The overall greenish color of the second coherence RGB composite originates from the longer interval for the $4^{\text {th }}$ interval (and related lower red values). The overall reddish color of the forth coherence RGB composite originates from the longer interval for the $11^{\text {th }}$ interval (and related lower blue values). Areas with very significant changes in the coherence levels are not observed much, at least in this area. Over the urban part the coherence remains high for all intervals considered.

\section{CONCLUSIONS}

The differential interferograms generated confirm that S1 IWS data can be used for interferometry. The examples confirm that it is possible to comply with the very high image co-registration accuracy requirements necessary to achieve seamless interferometric phases and coherence maps without anomalies at the interfaces between subsequent bursts and between sub-swaths. Large area S1 IWS coherence products can be generated, thanks to the overall swath width of about $250 \mathrm{~km}$ of the IWS acquisitions.

The potential of S1 IWS data for landuse classification and for monitoring temporal change is as expected for $\mathrm{C}$-band data with an overall similar spatial resolution as ERS and ENVISAT and with 12-day repeat intervals. This means on one hand that the use of the TOPS mode did not introduce anomalies or degradations as compared to stripmap mode data. On the other hand it also means that a significant part of the potential identified with ERS-1 3-day data and with ERS-1/2 Tandem interferometry depends on the short 1 and 3 day intervals. The 12-day intervals are significantly longer and as a consequence the coherence over low vegetation is significantly lower and therefore the contrast to forest is much reduced. Investigations should focus on the 6-days interferograms possible with
Sentinel-1B starting in early 2016

\section{ACKNOWLEDGEMENT}

ESA is acknowledged for providing the Sentinel-1 data. This work was supported by the EC FP7 Projects CRYOLAND and SEN3APP.

\section{REFERENCES}

[1] Wegmüller U. and C. L. Werner, SAR interferometric signatures of forest, IEEE Trans. Geosci. Remote Sensing, Vol. 33, No. 5, pp. 1153-1161, 1995.

[2] Askne J. and M. Santoro, "Automatic modelbased estimation of boreal forest stem volume from repeat pass C-band InSAR coherence," , IEEE Trans. Geosci. Remote Sensing, Vol. 47, 2, pp. 513-516, 2009.

[3] Wegmüller U. and C. L. Werner, Retrieval of vegetation parameters with SAR interferometry, IEEE Trans. Geosci. Remote Sensing, Vol. 35, No. 1, pp. 18-24, 1997.

[4] De Zan F. and A. Guarnieri, Terrain Observation by Progressive Scans, IEEE Trans. Geosci. Remote Sensing, vol. 44, no. 9, pp. 2353-2360, 2006.

[5] Prats P., L. Marotti and R. Scheiber, Investigation on TOPS Interferometry with TerraSAR-X, Proc. of IGARSS 2010.

[6] Scheiber R. and A. Moreira, Coregistration of interferometric SAR images using spectral diversity, IEEE Trans. Geosci. Remote Sensing, vol. 38, no. 5, pp. 2179-2191, 2000. 